\title{
EPIDEMIOLOGY OF DENGUE IN SRI LANKA BEFORE AND AFTER THE EMERGENCE OF EPIDEMIC DENGUE HEMORRHAGIC FEVER
}

\author{
WILLIAM B. MESSER, U. TISSA VITARANA, KAMALANAYANI SIVANANTHAN, JAYANTHI ELVTIGALA, \\ L. D. PREETHIMALA, R. RAMESH, NALINI WITHANA, DUANE J. GUBLER, AND ARAVINDA M. DE SILVA \\ Department of Microbiology and Immunology, University of North Carolina, Chapel Hill, North Carolina; Virology Department, \\ Medical Research Institute, Colombo, Sri Lanka; Division of Vector-Borne Infectious Diseases, National Center for Infectious \\ Diseases, Centers for Disease Control and Prevention, Fort Collins, Colorado
}

\begin{abstract}
Before 1989, dengue epidemiology in Sri Lanka was characterized by frequent transmission of all four dengue serotypes but a low incidence of dengue hemorrhagic fever (DHF). After 1989, cases of DHF dramatically increased. Here we present the results of epidemiologic studies conducted in Colombo, Sri Lanka before and after epidemic emergence of DHF in 1989. We compared the proportion of dengue cases among people with fever attending clinics from 1980 to 1984 and in 1997 and 1998 to determine if an increase in dengue transmission was associated with more DHF cases being reported. We also compared the relative distribution of dengue virus serotypes circulating in Colombo before and after the emergence of DHF. We detected no significant differences in dengue as a proportion of fever cases or in serotype distribution between the pre and post-DHF periods. We conclude that an increase in virus transmission or a change in circulating serotypes does not explain the epidemic emergence of DHF in Sri Lanka.
\end{abstract}

\section{INTRODUCTION}

Dengue viruses are mosquito-borne flaviviruses that have plagued people for centuries. ${ }^{1}$ Starting in the middle of the 20th century, large-scale urbanization and increasing human populations in tropical parts of the world created conditions especially favorable for dengue transmission. These changes led to the current global dengue pandemic, which is characterized by a dramatic increase in dengue infections and an expanding geographic distribution of both the viruses and the mosquito vector, Aedes aegypti. ${ }^{1}$

Most people infected with dengue viruses are asymptomatic or develop dengue fever (DF). The signs and symptoms of DF include high fever, myalgia, headache, vomiting, and loss of appetite. ${ }^{2,3}$ Although dengue fever is a serious, debilitating condition, it is usually not fatal. A minority of people infected with dengue viruses develop dengue hemorrhagic fever and dengue shock syndrome (DHF/DSS). The acute phase of DHF/DSS begins similar to that in DF but patients then abruptly develop a severe vascular permeability syndrome that may lead to shock and death., ${ }^{2,3}$

The distribution of DHF/DSS in Asia has been particularly enigmatic. Before 1989, DHF was common in Southeast Asia but rare in the Indian subcontinent despite hyperendemic transmission of dengue viruses in both regions. ${ }^{1,4}$ In 1989, this pattern of severe disease changed and regular epidemics of DHF have been reported from several countries in the Indian subcontinent. ${ }^{1,4}$ Here we report the results of epidemiologic studies that were done to understand factors responsible for the recent emergence of epidemic DHF in Sri Lanka.

There are four distinct serotypes of dengue virus, designated DEN-1, DEN-2, DEN-3, and DEN-4. Infection with one serotype confers immunity to that serotype but not to heterologous serotypes. ${ }^{5}$ Both retrospective and prospective studies have demonstrated that a person experiencing a secondary dengue infection is at a greater risk of developing DHF/DSS than a person experiencing a primary infection. ${ }^{6,7}$ To explain the increased risk of severe disease with secondary infection, Halstead has proposed that cross-reactive antibody from the primary infection enhances a secondary infection by binding to virus and increasing uptake into FC receptorbearing target cells of the monocytic/ macrophage lineage. ${ }^{8,9}$
More recently, this antibody-dependent enhancement (ADE) model has been further expanded to include roles for cellular immunity and host cytokines and chemical mediators in the pathogenesis of DHF. ${ }^{10}$

However, ADE alone does not sufficiently explain severe disease because DHF is observed in primary cases, and not all secondary cases develop DHF. ${ }^{11-17}$ Epidemiologic studies point to particular dengue virus strains being more virulent than others. ${ }^{16-19}$ Historically the dengue genotypes endemic to Central and South America have caused mild disease, while the introduction of Asian genotypes to the region has led to DHF epidemics. ${ }^{20-23}$ Similarly, outbreaks of DHF in some Pacific islands have been traced to the introduction of Southeast Asian dengue strains. ${ }^{24}$ The current consensus in the field is that certain virulent genotypes cause severe disease, most often, in the context of a secondary infection. ${ }^{7,19}$

To further understand the interplay between viral strains and secondary infections in severe disease, we have studied the epidemiology of dengue in Colombo, Sri Lanka where DHF has emerged as a serious public health threat relatively recently. The Indian subcontinent, where all four virus serotypes are present, did not have regular epidemics of $\mathrm{DHF} /$ DSS before 1989. Small outbreaks occurred in India in 1963 and in Sri Lanka in 1965-1966. ${ }^{4}$ In the Sri Lankan 1965-1966 epidemic, 26 cases of DHF/DSS were documented with six deaths (Medical Research Institute [MRI], Colombo, Sri Lanka, unpublished data). DEN-1 and DEN-2 viruses were isolated from the outbreak. Thereafter, DHF cases were only reported sporadically ( $<10$ cases/year) from Sri Lanka until 1989 when the incidence of DHF abruptly increased, with 206 hemorrhagic cases being reported. ${ }^{4,25}$ Since 1989, DHF rates have averaged more than 800 cases per year (Figure 1). ${ }^{26} \mathrm{~A}$ similar increase in the number of DHF cases was reported from several major Indian cities in the 1990s. ${ }^{4,27}$ The 1990s saw a long-term shift in the pattern of dengue disease in the Indian subcontinent, and the region now has regular epidemics of DHF/DSS.

To understand the sudden and long-term increase in DHF cases in Sri Lanka, we have compared the epidemiology of dengue infections before (1980-1984) and after (1997-1998) epidemic DHF emergence in Colombo, Sri Lanka. Specifically, we present data on three aspects of dengue epidemiol- 
DHF cases in Sri Lanka (Source: MRI and Epidemiology Unit, Colombo)

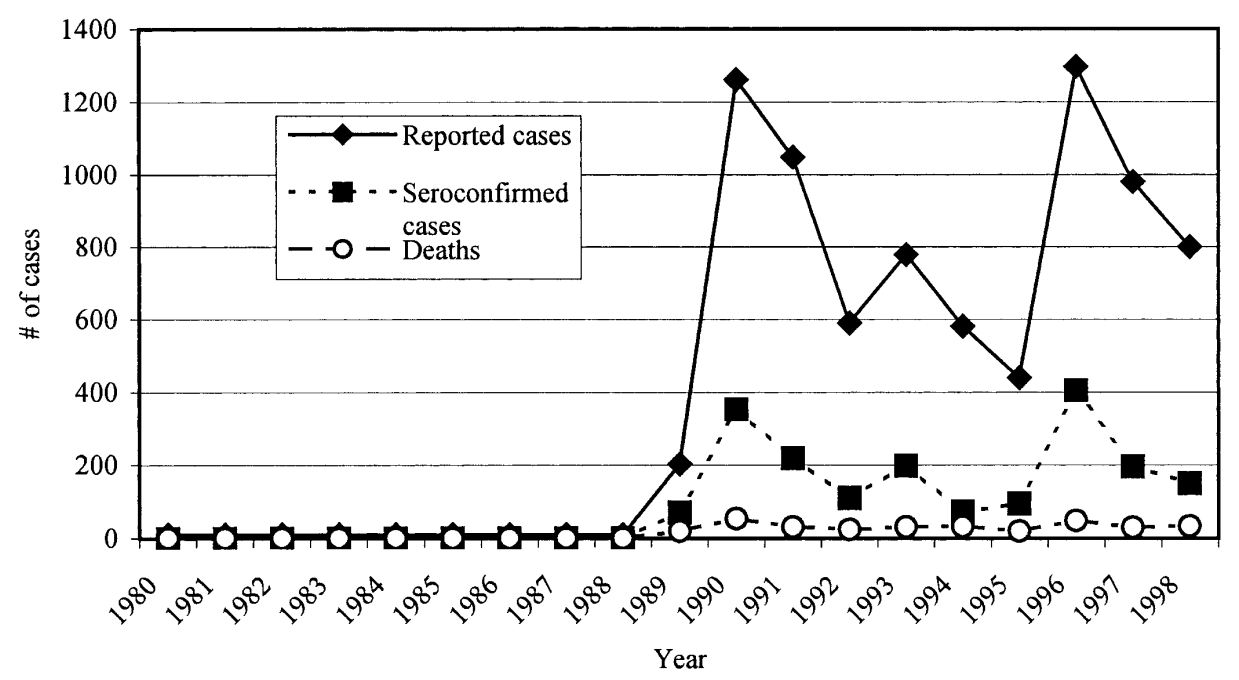

Figure 1. Cases of dengue hemorrhagic fever (DHF) in Sri Lanka, 1980-1998. The data are based on cases reported to the Epidemiology Unit of the Ministry of Health and cases tested at the Medical Research Institute (MRI) in Colombo. The case definition for DHF consists of at least fever, hemorrhagic symptoms, and a platelet count $<100,000 / \mathrm{mm}^{3}$. Serologic confirmation was based on the hemagglutination inhibition test.

ogy: 1) the proportion of dengue cases among people with fever attending primary care clinics, 2) the ratio of primary and secondary dengue infections among dengue cases in both clinics and hospital wards, and 3) the relative abundance of dengue serotypes isolated from DF cases. Our data demonstrate that the intensity of dengue transmission and the relative abundance of each serotype have remained stable after the emergence of DHF. The reason for the emergence of DHF in Sri Lanka is more complex than a simple increase in the intensity of virus transmission or the displacement of one serotype by a different serotype.

\section{MATERIALS AND METHODS}

Study site. Sri Lanka is an island with an area of $65,000 \mathrm{~km}^{2}$ (436 km long and $225 \mathrm{~km}$ wide) that is located $33 \mathrm{~km}$ south of India. The current study was conducted in the coastal city of Colombo, which is located on the southwestern side of the island. Colombo is the largest city in the country with a population of approximately 600,000 in 1980-1985 and nearly one million in 1998. The average annual rainfall in Colombo is 2,240 mm, with the bulk of the rain falling from May to August (southwest monsoon) and less from November to January (northeast monsoon). The average temperature ranges between 25 and $30^{\circ} \mathrm{C}$.

Prospective study among school children in Colombo (1980-1985). Nine schools located within walking distance from the municipal clinics were selected to enroll students for the prospective school cohort study. Blood samples were taken from primary school children (5-7 years old). Each cohort of 5-7-year-old children was bled in October and six months later in April of the following year. Five cohorts were tested from 1980 to 1985 . Blood was obtained by finger stick and collected onto two fully saturated filter paper discs (1.0 $\mathrm{cm}$ diameter) and into five microcapillary tubes. The filter paper discs were air-dried, stored in a freezer $\left(-10^{\circ} \mathrm{C}\right.$ to $\left.20^{\circ} \mathrm{C}\right)$, and tested as pairs (six months apart) by the hemagglutina- tion inhibition (HI) test to determine seroconversion rates. The ethical clearance committee of the MRI in Colombo, Sri Lanka approved the protocol for collecting blood for the school cohort study.

Surveillance of patients with fever attending clinics (1980-1984). For the fever surveillance study, five study areas were chosen to obtain proper geographic, ethnic, and socioeconomic representation of the city population. Two of the areas were located in the northern part of the city, two in the central part, and one in the southern part. These study areas covered 24 of the 47 municipal wards in Colombo. In each of the five study areas, a municipal clinic and a private practitioner clinic were selected for case enrollment by a team consisting of a physician, nurse and public health inspector. Each day of the week (Monday-Friday) was devoted to enrolling patients at the clinics in one study area so that over the fiveday week patients were enrolled from all five study areas. In addition to the municipal clinics, the outpatient clinic at the Lady Ridgeway Children's Hospital (LRH) was also visited weekly to enroll patients. Only patients with a fever of unknown origin (no respiratory, diarrheal, or other obvious cause for the fever) were enrolled in the study. A clinical history was obtained and a physical examination was performed on each patient enrolled in the study. A venous blood sample was taken from each patient and the samples were taken to the MRI in a vacuum flask on wet ice at the end of the morning's work. A convalescent blood sample was collected from most patients two weeks later by appointment at the clinic or by going to the patient's home. Records for which patient data were incomplete were excluded from all analyses. The ethical clearance committee of the MRI in Colombo, Sri Lanka approved the protocol for dengue surveillance at clinics. These two early studies were designed and conducted by two of the co-investigators (UTV and DJG).

Surveillance of patients with fever attending the LRH outpatient clinic (1997-1998). Fever cases were enrolled between July 4, 1997 to August 8, 1997 and again from December 17, 
1997 to January 26,1998 . This study is collectively referred to as the 1997-1998 clinic study. The protocol for enrolling patients was similar to the 1980-1984 clinic study except for the following changes: patients were only enrolled at the LRH outpatient clinic, and the study was restricted to children between the ages of one and 12. Researchers visited the clinic daily from 9:00 AM to noon to collect sera from patients presenting with a dengue-like illness for four or fewer days. To obtain convalescent samples, the patients were asked to return at least six days after the onset of fever. The time interval between acute and convalescent samples was reduced in the post DHF study because an IgM enzyme-linked immunosorbent assay (ELISA) and a reverse transcriptase-polymerase chain reaction (RT-PCR) assay were used in addition to the $\mathrm{HI}$ test in diagnosing dengue infections. Records for which patient data were incomplete were excluded from statistical analyses. The ethical clearance committee of the MRI in Colombo, Sri Lanka approved the protocol for dengue surveillance at the LRH clinic.

Surveillance of suspected DHF patients admitted to the LRH wards (1997-1998). Data for this study were collected between August 5, 1997 and August 14, 1997 and from July 13, 1998, to August 20, 1998. Children suspected of having DHF were admitted to six wards at the LRH. Suspected DHF cases are referred from the LRH outpatient clinic as well as other government and private clinics in Sri Lanka. The MRI staff visited all the medical wards at the LRH every day to collect blood from suspected DHF cases. An acute sample was collected as soon as possible after admission and a second sample was collected on the day of discharge from hospital. The patients' charts were read and the attending physicians were consulted to record the clinical and physical findings as well as the clinical laboratory test results. Records for which patient data were incomplete were excluded from all analyses.

Hemagglutination inhibition test. Only paired samples were analyzed from the 1980-1984 study. Samples were tested by the HI test using the method of Clarke and Casals modified for microtiter plates. ${ }^{28}$ All antigens were prepared from mouse brain infected with prototype viruses and extracted with sucrose-acetone. Nonspecific inhibitors in sera were removed by extraction with kaolin, and agglutinins were removed by extraction with goose red blood cells. The antigens used in the HI test were obtained from two dengue serotypes (serotype 1 and 2, or 3 and 4), Japanese Encephalitis virus, and Sindbis virus. The samples were tested at two-fold dilutions up to a dilution of 1:2,560. The HI test was performed as described above for the 1997-1998 samples except that Japanese encephalitis and Sindbis antigen were not used.

Isolation of virus. All samples from 1980-1985 and 1989-1996 were sent to the Dengue Branch of the Centers for Disease Control and Prevention (CDC) in San Juan, Puerto Rico for virus isolation by the mosquito inoculation technique using adult Aedes aegypti and/or Toxorhynchites amboinen$\operatorname{sis}^{29}$ or infection of C6/36 cells grown in Eagle's minimum essential medium supplemented with $5 \%$ fetal calf serum. ${ }^{30}$ Serum samples, stored at $-70^{\circ} \mathrm{C}$, were thawed and inoculated intrathoracically into mosquitoes undiluted and at dilution of 1:5 in phosphate-buffered saline (PBS) containing 20\% heat-inactivated calf serum. Inoculated mosquitoes were held at $30^{\circ} \mathrm{C}$ for 14 days, killed by freezing, and stored at $-70^{\circ} \mathrm{C}$ until tested for virus. Mosquito brain squashes were examined by the direct fluorescent antibody (DFA) test for the presence or absence of viral antigen using a conjugate prepared from high-titered human serum. ${ }^{30}$ The C6/36 cells were infected with $50 \mu \mathrm{L}$ of undiluted acute serum ( $<5$ days) and incubated for seven days at $32^{\circ} \mathrm{C}$. Cells were fixed to slides with cold buffered acetone and infecting virus was identified by DFA followed by IFA using serotype-specific monoclonal antibodies $^{30}$ and/or an RT- PCR. ${ }^{31}$

RT-PCR. Virus was detected and identified by RT-PCR using the method developed by Lanciotti and others ${ }^{31}$ Briefly, RNA was extracted by the guanidine isothiocyanate method or using a commercially available RNA isolation kit (viral isolation kit; Qiagen, Valencia, CA). Target viral RNA was converted to cDNA by reverse transcriptase Rous-associated virus-2 and consensus downstream primer D2. The cDNA was amplified by a nested PCR using Taq polymerase and consensus primers D1 and D2 in the first round of amplification and replacing primer D1 with serotype-specific primers TS1-TS4 in the second round of amplification. Second-round PCR products were visualized and analyzed by electrophoresis on agarose gels stained with ethidium bromide.

IgM ELISA. Samples collected in the 1997-1998 studies were tested by IgM capture ELISA. ${ }^{32}$ For the IgM ELISA, 96-well plates were coated with goat anti-human IgM antibody, to which serum samples diluted 1:40 were added, followed by a dengue antigen mixture (D1, 2, 3, 4) and horseradish peroxidase (HRP)-conjugated anti-flavivirus monoclonal antibody. After addition of HRP substrate, sera were scored as positive for infection if the optical density at $405 \mathrm{~nm}$ $\left(\mathrm{OD}_{405}\right)$ was $\geq 0.2$.

IgG ELISA. Samples collected in the 1997-1998 ward study were tested by IgG ELISA using a modification of the method described by Chungue and others. ${ }^{33}$ Briefly, hyperimmune ascites fluid (anti D1-D4) was added to each well of 96-well plates and incubated overnight at $4{ }^{\circ} \mathrm{C}$. Plates were washed three times with PBS, blocked with standard diluent, and incubated for one hour at $37^{\circ} \mathrm{C}$, followed by the addition of a D1-D4 antigen mixture and incubation at $37^{\circ} \mathrm{C}$ for an additional hour. After the plates were washed three times in PBS, a 1:40 diluted serum was added to the first well and four-fold dilutions were carried out to the eighth well. Plates were incubated for one hour at $37^{\circ} \mathrm{C}$, washed three times with PBS, followed by the addition of anti-human $\mathrm{IgG}$ conjugated to HRP, incubation at room temperature for one hour, and subsequent addition of HRP substrate. Color development was continued at room temperature until a low positive control reached an $\mathrm{OD}_{405}$ of 0.15 in the third or fourth dilution, at which point the plates were read.

Classification of dengue infections: 1980-1985 school cohort study. In the cohort study, a titer of 1:20 for both dengue antigens, or 1:40 for one, was taken to indicate a past infection. However, when a serum had titers $<1: 40$ for dengue, Sindbis, and Japanese encephalitis viruses, they were not considered positive since they were thought to be non-specific. A new dengue infection occurring during the period of this study was indicated by a four-fold or greater increase in titer or a conversion from negative to positive against at least two dengue antigens.

Classification of dengue infections: 1980-1984 clinic study. Current dengue infections were diagnosed by a four-fold or greater increase in $\mathrm{HI}$ antibody titers between acute and convalescent serum samples against any of the four dengue an- 
tigens. An infection was defined as primary when there was no HI antibody detectable in the acute serum taken four days or less after onset of illness and the convalescent titer was $\leq 1: 640$. Secondary dengue infection was diagnosed when the convalescent titer was $\geq 1: 1,280$ against at least one antigen. ${ }^{34}$ Secondary infections were also diagnosed when there was detectable $\mathrm{HI}$ antibody in the acute serum sample and a fourfold increase in the convalescent sample against two dengue antigens. Samples that did not conform to these criteria were classified as indeterminate and excluded from statistical analyses.

Classification of dengue infections: 1997-1998 clinic and ward studies. Both paired and single serum samples were tested with a variety of tests. Acute samples (four or fewer days of fever) were tested by IgM ELISA, RT-PCR, virus isolation, and HI test or IgG ELISA. Convalescent samples were only tested by IgM ELISA and HI test. Samples were scored as positive if 1) they were RT-PCR positive, 2) they were IgM positive, 3) they had a four-fold increase in HI titer between acute and convalescent samples, or 4) virus was isolated from the sample. For dengue-positive samples, if the acute sample ( $\leq 4$ days after onset) had an HI titer $\geq 20$ (IgG $>160$ ), it was defined as a secondary infection. In denguepositive cases for which a second serum sample ( $\geq 5$ days) was available, an HI titer $\geq 1,280$ (IgG ELISA titer $\geq 40,960$ ) was considered to represent a secondary infection regardless of the acute phase titer. Samples that did not conform to these criteria were classified as indeterminate and excluded from statistical analyses. Disease severity for dengue cases was defined as follows: 1) Dengue fever with hemorrhage if the patient had signs of hemorrhage or a platelet count less than $100,000 / \mathrm{mm}^{3}$ and 2) DHF if the patient had signs of hemorrhage, evidence of plasma leakage, and a platelet count less than $100,000 / \mathrm{mm}^{3}$. Dengue cases without any hemorrhagic symptoms and with platelet counts greater than $100,000 / \mathrm{mm}^{3}$ were classified as DF cases.

Statistical analyses. Data were entered, sorted, and analyzed by Access ${ }^{\circledR}$ (Microsoft, Redmond, WA). Chi-square, analysis of variance, Tukey's honestly significant data test, Dunnett's test ${ }^{35}$ odds ratios, and confidence intervals were calculated using SAS and JMP (SAS Institute, Cary, NC), SPSS (SPSS, Inc., Chicago, IL), or Excel ${ }^{\circledR}$ (Microsoft) software. The level of significance for all statistical comparisons was $<0.05$. Age adjustments of clinic surveillance data were done using three strata of cases: $1-4$ years old, 5-8 years old, and 9-12 years old. The standard population was the summed 1980-1984 population in each strata. Adjustment of proportions and ratios was performed using standard methods as described by Rothman and Sander. ${ }^{36}$ Dunnett variances were weighted by total count for each of the years compared.

\section{RESULTS}

Prospective school cohort study. The data from the prospective school cohort study were analyzed to determine the dengue seroprevalence and six-month infection rate among 5-7-year-old school children. In all, 7,270 children were bled over the five-year period, averaging 1,454 per year (range = 1,004-1,949) (Table 1). At the time of enrollment, the dengue seroprevalence in each cohort averaged $50 \%$, indicating frequent exposure of this population to dengue viruses. The sixmonth infection rate averaged $15.6 \%$ (range $=10.8-19.4 \%$ ), but in most years the rate was higher than the average (Table 1). The number of primary infections exceeded secondary infections in every year, and for the five years of the study, $63.4 \%$ were classified as primary and $36.6 \%$ as secondary (Table 1). These results establish intense dengue transmission and frequent exposure to secondary infections among school children in Colombo from 1980 to 1985.

Clinic and ward studies: demographic characteristics. The mean ages and sex ratios of people enrolled from 1980 to 1984 and from 1997 to 1998 are shown in Table 2. The 1980-1984 study population had an age range of 0-59 years, and showed an increasing mean age from 11.17 years in 1980-1981 to 14.44 years in 1983-1984. The difference between these two means was statistically significant $(P<0.05)$. The overall ratio of males to females was 0.90 , with more females than males enrolled every year except 1982-1983. However, the male to female ratios were not statistically different from year to year. Because the 1997-1998 data were collected at a pediatric hospital, the mean ages (clinic $=5.35$ years and ward $=6.06$ years) were considerably lower than the mean age (12.60) of people enrolled between 1980 and 1984, which included both adults and children (Table 2).

Clinic dengue fever surveillance, 1980-1984. An average of 248 (range $=137-319$ ) cases per year of fever of unknown origin (FUO) were studied in five regions of Colombo (Table 3). Overall, $15.96 \%$ were confirmed as dengue infections, with confirmation rates ranging from a low of $12.30 \%$ (1981-1982) to a high of $20.44 \%$ (1983-1984). Among the dengue-positive cases, the number of secondary infections $(65.56 \%)$ exceeded the number of primary infections $(27.15 \%)$, with the number of secondary infections exceeding the number of primary infections in every complete year (Table 3 ). There were only 33 documented cases with fever and hemorrhage admitted to Colombo hospitals during the five years of the study. Of the 33 cases, $11(33 \%)$ were laboratory confirmed as having dengue infection, 10 were secondary infections, and one was a primary infection. None of the cases conformed to the World Health Organization (WHO) case definition for DHF.

Clinic dengue fever surveillance, 1997-1998. A total of 166

TABLE 1

Dengue infections among school children, in Colombo, Sri Lanka, 1980-1985*

\begin{tabular}{|c|c|c|c|c|}
\hline \multirow{2}{*}{$\begin{array}{c}\text { Year } \\
\text { (Oct-Sept) }\end{array}$} & \multirow{2}{*}{$\begin{array}{l}\text { Total } \\
\text { tested }\end{array}$} & \multicolumn{3}{|c|}{ Seroconversions } \\
\hline & & Total new (\%) & Primary $(\%)$ & Secondary (\%) \\
\hline 1980-1981 & 1,230 & $228(18.54)$ & $127(55.70)$ & $101(44.30)$ \\
\hline 1981-1982 & 1,505 & $252(16.74)$ & $152(60.32)$ & $100(39.68)$ \\
\hline $1982-1983$ & 1,939 & 209 (10.78) & $142(67.94)$ & $67(32.06)$ \\
\hline 1983-1984 & 1,592 & $270(16.96)$ & $205(75.93)$ & 65 (24.07) \\
\hline 1984-1985 & 1,004 & 164 (16.33) & $86(52.44)$ & $78(47.56)$ \\
\hline Total & 7,270 & $1,123(15.58)$ & $712(63.40)$ & $411(36.60)$ \\
\hline
\end{tabular}


TABLE 2

Summary of clinic and ward study populations mean ages and sex ratios

\begin{tabular}{|c|c|c|c|c|c|c|}
\hline \multirow[b]{2}{*}{$\begin{array}{c}\text { Year } \\
\text { (Nov-Oct) }\end{array}$} & \multicolumn{3}{|c|}{ Clinic cases } & \multicolumn{3}{|c|}{ Ward cases } \\
\hline & $\begin{array}{c}\text { Enrolled } \\
\text { cases }\end{array}$ & $\begin{array}{l}\text { Age } \dagger \text { mean } \\
\text { (range) }\end{array}$ & $\begin{array}{c}\text { Sex ratio末 } \\
\text { (M:F) }\end{array}$ & $\begin{array}{c}\text { Enrolled } \\
\text { cases }\end{array}$ & $\begin{array}{c}\text { Age, mean } \\
\text { (range) }\end{array}$ & $\begin{array}{l}\text { Sex ratio } \\
(\mathrm{M}: \mathrm{F})\end{array}$ \\
\hline 1980-1981 & 238 & $11.17^{\mathrm{A}}(0-46)$ & $0.71(99: 139)$ & NA* & NA & NA \\
\hline $1981-1982$ & 252 & $12.03^{\mathrm{AB}}(0-55)$ & $0.92(121: 131)$ & NA & NA & NA \\
\hline $1982-1983$ & 319 & $13.31^{\mathrm{B}}(1-50)$ & $1.03(162: 157)$ & NA & NA & NA \\
\hline 1983-1984 & 137 & $14.44^{\mathrm{B}}(1-59)$ & $0.96(67: 70)$ & NA & NA & NA \\
\hline Total (1980-1984) & 946 & $12.60(0-59)$ & $0.90(449: 497)$ & NA & NA & NA \\
\hline 1997-1998 & 166 & $5.35(1-12)$ & $0.95(81: 85)$ & 87 & $6.06(1-12)$ & $0.67(35: 52)$ \\
\hline
\end{tabular}

- NA = not applicable. mean ages were not compared to 1980-1984 mean ages because the 1980-1984 study included children and adults whereas the 1997-1998 study only included children 1-12 years old.

$\ddagger$ The ratios of males to females were not significantly different for any of the clinic study populations.

FUO cases were studied, of which $41(24.70 \%)$ were dengue infections (Table 3). The number of primary infections $(56.10 \%)$ exceeded the number of secondary infections $(34.15 \%)$ in the 1997-1998 clinic study (Table 3).

Ward dengue surveillance, 1997-1998. Eighty-seven suspected DHF cases admitted to the LRH were followed; 51 $(58.62 \%)$ were confirmed as dengue infections. Among the hospitalized cases, the number of secondary infections $(62.75 \%)$ exceeded the number of primary infections (19.60\%) (Table 3).

Comparison of clinic surveillance data from 1980-1984 and 1997-1998. One plausible explanation for the emergence of epidemic DHF in 1989 may be an overall increase in dengue virus transmission, leading to more frequent exposure of people to dengue infections, and ultimately more frequent exposure to secondary infections. However, true incidence data are not available for the post-DHF years. To detect difference in transmission intensity before and after the emergence of epidemic DHF, we compared the proportion of people with dengue among FUO clinics and the ratios of secondary to primary infections in dengue cases among clinic FUO cases in 1980-1984 and 1997-1998.

The study populations in 1997-1998 were limited to children 1-12 years old, while the 1980-1984 study population included individuals $0-59$ years old. Furthermore, the mean age increased over the course of the 1980-1984 study (Table 2) and the stratified age structures were found to be different (by chi-square test). Therefore, age is likely to confound comparisons within the 1980-1984 study and between the 1980-1984 and 1997-1998 study populations. The data were age adjusted as detailed in the Materials and Methods section.
The unadjusted proportion of dengue cases among children with fever ranged from $10.37 \%$ in $1981-1982$ to a high of $19.72 \%$ in $1983-1984$ compared with $22.29 \%$ in $1997-1998$ (Table 4). Following age adjustment, the expected proportion of dengue cases ranged from $9.76 \%$ in $1981-1982$ to $18.31 \%$ in 1983-1984; in 1997-1998 it was $18.80 \%$ (Table 4). While the proportions show considerable variability, only 1981-1982 was significantly less than that of 1997-1998 (by Dunnett's test). An age-standardized secondary to primary infection ratio was also calculated for each study year. The age adjusted ratios of secondary to primary infections for the 1980-1984 study period ranged from 0.5 in 1981 to 2.53 in 1982 , while in 1997-1998 the ratio of secondary to primary infections was 0.75 (Table 4).

Relative distribution of dengue serotypes among patients. Disease severity may have changed in Sri Lanka because of changes in the abundance of a particular dengue serotype in 1989. We compared the relative abundance of each serotype before and after 1989. From the dengue cases identified from the clinic study conducted from 1980 to 1985, virus was isolated from 56 specimens (Table 5). The relative abundance of the four serotypes had a distinct pattern, which was dominated by D2 and D3, with D1 and D4 found in considerably lower abundance. Specifically, D2 was predominant (23 isolates), followed by D3 (20 isolates) and D1 (12 isolates) (Table 5). D3 was isolated in all five years of the study, D2 in the first four years, and D1 in three years. Although D4 was not isolated from any of the cases during the study period, this serotype was isolated from a U.S. embassy resident in Sri Lanka in 1981.

Acute serum samples collected in 1989, 1990, 1992, 1994,

TABLE 3

Positive dengue cases in clinic and ward study

\begin{tabular}{|c|c|c|c|c|c|}
\hline \multirow[b]{2}{*}{$\begin{array}{c}\text { Year } \\
\text { (Nov-Oct) }\end{array}$} & \multirow[b]{2}{*}{$\begin{array}{l}\text { Enrolled } \\
\text { cases }\end{array}$} & \multicolumn{4}{|c|}{ Positive for dengue* } \\
\hline & & $\begin{array}{l}\text { Total cases } \\
(\%)\end{array}$ & $\begin{array}{l}\text { Primary } \\
(\%)\end{array}$ & $\begin{array}{c}\text { Secondary } \\
(\%)\end{array}$ & $\begin{array}{c}\text { Indeterminates } \\
(\%) \dagger\end{array}$ \\
\hline 1980-1981 & 238 & 38 (15.97) & $9(23.68)$ & $25(65.79)$ & $4(10.53)$ \\
\hline 1981-1982 & 252 & 31 (12.30) & $10(32.26)$ & $17(54.84)$ & $4(12.90)$ \\
\hline 1982-1983 & 319 & 54 (16.93) & $12(22.22)$ & $39(72.22)$ & $3(5.56)$ \\
\hline 1983-1984 & 137 & $28(20.44)$ & $10(35.71)$ & $18(64.29)$ & $0(0.00)$ \\
\hline Total & 946 & $151(15.96)$ & $41(27.15)$ & 99 (65.56) & $11(7.23)$ \\
\hline 1997-1998 clinic & 166 & $41(24.70)$ & $23(56.10)$ & $14(34.15)$ & $4(9.76)$ \\
\hline 1997-1998 ward & 87 & $51(58.62)$ & $10(19.60)$ & $32(62.75)$ & $9(17.64)$ \\
\hline
\end{tabular}

* See Materials and Methods for details of how cases were identified as dengue cases and how dengue cases were classified as primary and secondary.

$\dagger$ Indeterminates are dengue-positive cases that could not be classified as primary or secondary. 
TABLE 4

Comparison of clinic data from 1980-1984 and 1997-1998 studies*

\begin{tabular}{|c|c|c|c|c|c|c|c|}
\hline \multirow[b]{2}{*}{ Year } & \multirow[b]{2}{*}{$\begin{array}{l}\text { Enrolled fever } \\
\text { cases }\end{array}$} & \multicolumn{6}{|c|}{ Positive for dengue } \\
\hline & & $\begin{array}{l}\text { Total dengue } \\
\text { cases }\end{array}$ & $\begin{array}{l}\text { Crude } \\
\text { proportion }\end{array}$ & $\begin{array}{l}\text { Age adjusted } \\
\text { proportion }\end{array}$ & $\underset{(\%)}{\text { Primary }}$ & $\begin{array}{l}\text { Secondary } \\
(\%)\end{array}$ & $\begin{array}{c}\text { Adjusted ratio } \\
\text { (secondary:primary) }\end{array}$ \\
\hline 1980-1981 & 157 & 23 & 14.65 & 13.38 & $7(30.43)$ & $16(69.57)$ & 2.21 \\
\hline $1981-1982 \dagger$ & 164 & 17 & 10.37 & 9.76 & $12(70.58)$ & $5(29.42)$ & 0.50 \\
\hline $1982-1983$ & 191 & 29 & 15.18 & 13.61 & $8(27.59)$ & $21(72.41)$ & 2.53 \\
\hline 1983-1984 & 71 & 14 & 19.72 & 18.31 & $7(50.00)$ & $7(50.00)$ & 1.00 \\
\hline Total (1980-1984) & 583 & 83 & 19.24 & NA & $34(30.96)$ & $49(59.04)$ & 1.44 \\
\hline 1997-1998 & 166 & 37 & 22.29 & 18.80 & $23(62.16)$ & $14(37.84)$ & 0.75 \\
\hline
\end{tabular}

* Cases are for patients 1-12 years old. Age-adjusted proportions and ratios were calculated as described in the Materials and Methods. NA = not applicable.

$\dagger$ Different from the 1997-1998 proportion by Dunnett test $(P<0.05)$.

1997, and 1998 in Sri Lanka were sent to the Dengue Branch of CDC in Puerto Rico for virus isolation by mosquito inoculation or $\mathrm{C} 6 / 36$ tissue culture, both followed by IFA. Virus was isolated from 24 samples. An additional 86 serum samples collected in 1997 and 1998 were screened by RT-PCR and C6/36 tissue culture at the MRI in Colombo. As with the 1980-1985 samples, the distribution of serotypes isolated from 1989 to 1998 (Table 5) had a distinct pattern, with dengue 2 predominating (17 isolates), followed by dengue 3 (13 isolates) dengue 1 (10 isolates), and dengue 4 (2 isolates). D2 and D3 were isolated in all years except 1992, D4 was isolated only in 1992, and D1 was isolated in 1992, 1997, and 1998. The relative distribution of serotypes isolated from 1980 to 1985 was not significantly different from the relative distribution of serotypes isolated from 1989-1998 $(P>0.05$, by chi-square test). Rather, the relative distributions are remarkably similar.

Risk of severe disease with secondary infection. To test whether secondary infections are associated with an increased risk of developing severe disease in Sri Lanka following the emergence of epidemic DHF, we compared the proportion of primary to secondary infections among dengue patients with no signs of bleeding (DF) and dengue patients with signs of hemorrhage (DF plus hemorrhage and DHF cases) (Table 6). These values gave odds ratios of 0.24 and 4.25 of a severe disease being associated with a primary or secondary infection, respectively. These results suggest that once DHF emerged in Sri Lanka, secondary infections may be a risk factor for severe disease among people with symptomatic dengue infections. However, the finding is not statistically significant (overlapping confidence intervals), suggesting the need for further study with a larger number of cases.

\section{DISCUSSION}

Until now, the epidemiology of dengue infections in Sri Lanka has not been comprehensively described in the literature, although the country's unusual characteristic of a large number of secondary infections and absence of DHF prior to 1989 has been previously reported. ${ }^{36,37}$ The results presented here represent time periods from both before and after the emergence of epidemic DHF in Colombo, Sri Lanka, and make it possible to look for changes in the epidemiology of dengue transmission that correlate with the emergence of DHF.

Over the period from 1980 to 1985 only 11 cases of hemorrhagic disease were detected in Colombo and none of these met the WHO case definition for DHF. The LRH is the major children's hospital in the country, and provides free service that is used by both the rich and poor. Moreover, the specialist pediatricians there provide consultant services to private hospitals in Colombo. They were informed of the DF/DHF surveillance program, were aware of the dangers of DHF, and had been instructed in its clinical diagnosis and management. The outpatient staff at the LRH had also been instructed to admit all cases of fever with bleeding to the ward of the professor of pediatrics, who was participating in this study. Given the extent of awareness about dengue between 1980 and 1985, it is unlikely that epidemics of DHF occurred unnoticed by health authorities and on a scale similar to that that occurred after 1989.

The low number of DHF cases was not due to lack of exposure to dengue viruses. The prospective school cohort survey conducted from 1980 to 1985 demonstrated a seroprevalence of $50 \%$ and a six-month seroconversion rate of $15 \%$ among 5-7-year-old school children. The FUO clinic surveillance program also confirmed that dengue transmission and secondary infections were common in Colombo over this period, with secondary infections accounting for $59 \%$ of all dengue cases. Moreover, an outbreak with DEN-2 as the predominant virus serotype occurred in the fall of 1983. Despite a high rate of secondary infections, there were no DHF cases detected. ${ }^{38}$

The lack of DHF was not due to hypoendemicity. Multiple dengue virus serotypes (DEN-1, DEN-2, and DEN-3) circulated simultaneously in Colombo throughout most of the study period. Although dengue 4 was not isolated during 1980-1985 surveillance, there is evidence it was also circulating in Colombo from plaque reduction neutralization test assays on serum samples. ${ }^{38}$ If antibody dependent enhancement were the sole mechanism responsible for severe dengue disease, then one would have expected considerably more DHF in Colombo, Sri Lanka, from 1980 to 1985 given the intensity of transmission of multiple virus serotypes in Colombo over the period 1980-1985.

It is plausible that the DHF epidemics in 1989 started because the intensity of transmission increased above the levels observed in 1980-1984. Using dengue cases among children with FUO as a surrogate for dengue virus activity in Colombo, we did not find evidence for a large increase in virus activity following the emergence of epidemic DHF. Over the period 1980-1984, 10.37-19.72\% (Table 4) of FUO cases (unadjusted) were due to dengue, whereas in 1997-1998, the proportion was $22.29 \%$ (Table 4 ). Following age-adjustment, 
TABLE 5

Count of serotypes isolated before (1980-1985) and after (1989-1998) emergence of dengue hemorrhagic fever in Sri Lanka

\begin{tabular}{|c|c|c|c|c|c|}
\hline \multirow[b]{2}{*}{ Years } & \multicolumn{5}{|c|}{ Serotype } \\
\hline & $1 \underset{(\%)}{1 \text { Count }}$ & $2 \underset{(\%)}{2 \text { Count }}$ & $\begin{array}{c}3 \text { Count } \\
(\%)\end{array}$ & $\begin{array}{c}4 \text { Count } \\
(\%)\end{array}$ & Total \\
\hline 1980-1985 & $12(21.43)$ & $23(41.07)$ & $20(35.71)$ & $1(1.79)$ & 56 \\
\hline 1989-1998 & $10(23.81)$ & $17(40.48)$ & $13(30.95)$ & $2(4.76)$ & 42 \\
\hline Total & $22(22.45)$ & $40(40.82)$ & $33(33.67)$ & $3(3.06)$ & 98 \\
\hline
\end{tabular}

only the 1981-1982 proportion was significantly less than that of the 1997-1998 proportion of dengue cases. The 1997-1998 data were collected over relatively short periods (five weeks in 1997 and five weeks in 1998) and may not reflect dengue transmission over the entire year. In fact, the two five-week periods (July-August 1997 and December-January 1998) represent periods of high transmission because they coincide with the southwest and northeast monsoons, respectively. Therefore, the short sampling periods in 1997-1998 are more likely to overestimate than underestimate dengue activity in and around Colombo.

As a more stable estimate of intensity of dengue transmission, we also calculated an age-adjusted ratio of primary to secondary infections among children with dengue before and after the emergence of epidemic DHF (Table 4). The greater the ratio's value, the more intense dengue transmission is likely to be, yielding more secondary infections in a young (1-12-years old) population. The ratios of secondary to primary infections show considerable variation from one year to the next for the 1980-1984 study data, but only one year, 1981-1982, had a ratio less than 1 . A low value, 0.75 for $1997-$ 1998, suggests a lower intensity of transmission because fewer children were presenting with secondary infections. In fact, the data presented here suggest that the intensity of transmission may have decreased since DHF emergence, with only three of the four pre-DHF years having secondary to primary ratios greater than or equal to 1 . However, the statistical power to resolve such a change is lacking in this study. However, taken together, our results do not point to an overall increase in dengue transmission or the proportion of secondary infections following the emergence of epidemic DHF in Sri Lanka.

Finally, another possible explanation for the emergence of epidemic DHF in Sri Lanka could be an increase in the prevalence of a particular virus serotype. Dengue virus serotypes throughout the world have been correlated with epidemic DHF elsewhere, particularly in Thailand ${ }^{39,40}$ and Latin America with DEN-2, ${ }^{1,20,22,41}$ and Indonesia, ${ }^{34}$ the Americas, ${ }^{1,21}$ and the Pacific islands ${ }^{24}$ with DEN-3. While Sri Lanka

TABLE 6

Risk of severe disease with secondary infection*

\begin{tabular}{lcccc}
\hline \multicolumn{1}{c}{ Status } & DFhem + DHF & DF & Total & $\begin{array}{c}\text { Odds ratio } \\
(95 \% \text { CI })\end{array}$ \\
\hline Secondary & 17 & 29 & 46 & $4.25(0.75-23.9)$ \\
Primary & 4 & 29 & 33 & $0.24(0.04-1.35)$ \\
Total & 21 & 58 & 79 &
\end{tabular}

* The hemorrhagic (DFhem plus dengue hemorrhagic fever [DHF]) and dengue fever (DF) cases are from the 1997 and 1998 ward and clinic studies. The odds ratio compares the (DF) cases are from the 1997 and 1998 ward and clinic studies. The odds ratio compares the
risk of developing severe disease among symptomatic dengue cases with primary and secondary infections. CI = confidence interval. has had endemic circulation of all four virus serotypes, they do not occur in equal abundance. Therefore, shifts in the relative abundance of the serotypes could be correlated with changes in DHF. Clearly, DEN-1, DEN-2, and DEN-3 circulated throughout the period 1980-1985, and there is evidence that dengue 4 circulated as well. When compared with the distribution of isolates collected from 1989 to 1998, the two distributions are remarkably similar (Table 5). Far from suggesting that there has been a shift in the relative abundance of the four serotypes in Sri Lanka, these data suggest that the serotype distribution has remained stable through the emergence of DHF in Sri Lanka. The stability of the relative distribution of the virus serotypes further implies that dengue virus ecology in Sri Lanka, the collective interactions between the virus, its vector, human host, and the environment, may itself be relatively stable because the distribution of the virus serotypes has been maintained.

Previous studies have found a greater risk of hospitalization and severe disease with secondary infections compared with primary infections. ${ }^{13} \mathrm{We}$ tested whether secondary infections were a risk factor for severe disease during the 1997-1998 ward studies. We designated severe and mild disease based on the presence or absence of signs of hemorrhage in confirmed dengue cases. We did not use the WHO criteria alone for identifying severe disease cases because for many of the cases the hematocrit and platelet counts were not available. When cases were separated based on dengue infection with or without hemorrhagic manifestations, secondary infections were found to increase the odds of severe disease by 4.25 fold among people with symptomatic dengue infections. However, a study with a larger sample size is needed to confirm this association. Although secondary infections most likely contribute to DHF, the near absence of severe disease from 1980 to 1984 clearly demonstrates that some element other than immune enhancement is necessary for DHF to be present in a population.

The emergence of DHF in Sri Lanka was dramatic and persistent, suggesting that an equally dramatic and persistent change in the disease cycle occurred. The findings presented here indicate that no such change occurred in either virus serotype distribution, intensity of transmission, or proportion of secondary infections occurring in Colombo. Additionally, these findings lend credibility to the hypothesis first suggested by Rosen, Gubler, and others ${ }^{16-18}$ that virus strain is an important risk factor for DHF, and advanced by Lanciotti and others that small genotypic changes in dengue viruses are responsible for the DHF emergence in Sri Lanka. ${ }^{42}$ Such a change could be both dramatic and persistent but would be undetected by the epidemiologic approach used here. We are currently undertaking molecular genetic studies of dengue viruses isolated from DF and DHF patients in Sri Lanka over 
the study period 1980-1998. The results of those studies, which we hope to complete shortly, may provide more data on the genotype hypothesis.

Acknowledgments: We are extremely grateful to Jack Weiss (University of North Carolina at Chapel Hill Ecology Curriculum) for statistical advice, Vance Vorndam and the CDC Dengue Branch (San Juan, PR) for virus isolation and serology data, Sarah Williams and Amanda Brooks for data entry, and Dr. Gaya Colombage (Director of the MRI) and other staff members in the Virology Department at the MRI in Colombo for their help.

Authors' addresses: William B. Messer and Aravinda M. de Silva, Department of Microbiology and Immunology and Ecology Curriculum, University of North Carolina at Chapel Hill, CB\# 7290, Chapel Hill, NC 27599. U. Tissa Vitarana, Kamalanayani Sivananthan, Jayanthi Elvitigala, L. D. Preethimala, R. Ramesh, and Nalini Withana, Virology Department, Medical Research Institute, Danister de Silva Mawatha, Colombo, Sri Lanka. Duane J. Gubler, Division of VectorBorne Infectious Diseases, National Center for Infectious Diseases, Centers for Disease Control and Prevention, PO Box 2087, Fort Collins, CO 80522.

\section{REFERENCES}

1. Gubler DJ, 1997. Dengue and dengue hemorrhagic fever: its history and resurgence as a global public health problem. Gubler DJ, Kuno G, eds. Dengue and Dengue Hemorrhagic Fever. New York: CAB International, 1-22.

2. Nimmannitya S, 1987. Clinical spectrum and management of dengue haemorrhagic fever. Southeast Asian J Trop Med Public Health 18: 392-397.

3. Innis BL, 1995. Dengue and dengue hemorrhagic fever. Portersfield JS, ed. Exotic Viral Infections. London: Chapman and Hall, 103-146.

4. WHO, 2000. WHO Report on Global Epidemic-Prone Infectious Diseases. Geneva: WHO, Chapter 6.

5. Innis BL, 1997. Antibody responses to dengue virus infection. Gubler DJ, Kuno G, eds. Dengue and Dengue Hemorrhagic Fever. New York: CAB International, 221-244.

6. Halstead SB, 1990. Global epidemiology of dengue hemorrhagic fever. Southeast Asian J Trop Med Public Health 21: 636-641.

7. Halstead SB, 1997. Epidemiology of dengue and dengue hemorrhagic fever. Gubler DJ, Kuno G, eds. Dengue and Dengue Hemorrhagic Fever. New York: CAB International, 23-44.

8. Halstead SB, 1989. Antibody, macrophages, dengue virus infection, shock, and hemorrhage: a pathogenetic cascade. Rev Infect Dis 11: S830-S839.

9. Halstead SB, 1988. Pathogenesis of dengue: challenges to molecular biology. Science 239: 476-481.

10. Kurane I, Ennis FA, 1997. Immunopathogenesis of dengue virus infection. Gubler DJ, Kuno G, eds. Dengue and Dengue Hemorrhagic Fever. New York: CAB International, 273-290.

11. Carey DE, Myers RM, Reuben R, Rodrigues FM, 1966. Studies on dengue in Vellore, South India. Am J Trop Med Hyg 15: 580-587.

12. Woodall JP, Lopez-Correa RH, Sather GE, Moore CG, 1981. The absence of epidemic dengue hemorrhagic fever in the Americas. Hoota S, ed. Dengue Hemorrhagic Fever 1981. Proceedings of the First International Center for Medical Research Seminar. Kobe, Japan.

13. Burke DS, Nisalak A, Johnson DE, Scott RM, 1988. A prospective study of dengue infections in Bangkok. Am J Trop Med Hyg 38: 172-180.

14. Kuberski T, Rosen L, Reed D, Mataika J, 1977. Clinical and laboratory observations on patients with primary and secondary dengue type 1 infections with hemorrhagic manifestations in Fiji. Am J Trop Med Hyg 26: 775-783.

15. Eram S, Setyabudi Y, Sadono TI, Sutrisno DS, Gubler DJ, Sulianti Saroso J, 1979. Epidemic dengue hemorrhagic fever in rural Indonesia. II. Clinical studies. Am J Trop Med Hyg 28: 711-716.
16. Rosen L, 1977. The Emperor's New Clothes revisited, or reflections on the pathogenesis of dengue hemorrhagic fever. Am J Trop Med Hyg 26: 337-343.

17. Gubler DJ, Reed D, Rosen L, Hitchcock JR, Jr., 1978. Epidemiologic, clinical, and virologic observations on dengue in the Kingdom of Tonga. Am J Trop Med Hyg 27: 581-589.

18. Gubler DJ, Suharyono W, Lubis I, Eram S, Gunarso S, 1981. Epidemic dengue 3 in central Java, associated with low viremia in man. Am J Trop Med Hyg 30: 1094-1099.

19. Gubler DJ, 1998. Dengue and dengue hemorrhagic fever. Clin Microbiol Rev 11: 480-496.

20. Guzman MG, Kouri GP, Bravo J, Soler M, Vazquez S, Morier L, 1990. Dengue hemorrhagic fever in Cuba, 1981: a retrospective seroepidemiologic study. Am J Trop Med Hyg 42: 179-184.

21. Guzman MG, Vazquez S, Martinez E, Alvarez M, Rodriguez R, Kouri G, de los Reyes J, Acevedo F, 1996. Dengue in Nicaragua, 1994: reintroduction of serotype 3 in the Americas (in Spanish). Bol Oficina Sanit Panam 121: 102-110.

22. Rico-Hesse R, 1990. Molecular evolution and distribution of dengue viruses type 1 and 2 in nature. Virology 174: 479-493.

23. Rico-Hesse R, Harrison LM, Salas RA, Tovar D, Nisalak A, Ramos C, Boshell J, de Mesa MT, Nogueira RM, da Rosa AT, 1997. Origins of dengue type 2 viruses associated with increased pathogenicity in the Americas. Virology 230: 244-251.

24. Chungue E, Deubel V, Cassar O, Laille M, Martin PM, 1993. Molecular epidemiology of dengue 3 viruses and genetic relatedness among dengue 3 strains isolated from patients with mild or severe form of dengue fever in French Polynesia. $J$ Gen Virol 74: 2765-2770.

25. Vitarana T, 1990. Dengue haemorrhagic fever. Ceylon Med J 35: $83-87$.

26. Vitarana T, Jayakura WS, Withane N, 1997. Historical account of Dengue Hemorrhagic Fever in Sri Lanka. Dengue Bull 21: 117-118.

27. Srivastava VK, Suri S, Bhasin A, Srivastava L, Bharadwaj M, 1990. An epidemic of dengue haemorrhagic fever and dengue shock syndrome in Delhi: a clinical study. Ann Trop Paediatr 10: 329-334.

28. Mettler NE, Clarke DH, Casals J, 1971. Hemagglutination inhibition with arboviruses: relationship between titers and source of erythrocytes. Appl Microbiol 22: 377-379.

29. Rosen L, Gubler DJ, 1974. The use of mosquitoes to detect and propagate dengue viruses. Am J Trop Med Hyg 23: 1153-1160.

30. Gubler DJ, Kuno G, Sather GE, Velez M, Oliver A, 1984. Mosquito cell cultures and specific monoclonal antibodies in surveillance for dengue viruses. Am J Trop Med Hyg 33: 158-165.

31. Lanciotti RS, Calisher CH, Gubler DJ, Chang GJ, Vorndam AV, 1992. Rapid detection and typing of dengue viruses from clinical samples by using reverse transcriptase-polymerase chain reaction. J Clin Microbiol 30: 545-551.

32. Kuno G, Gomez I, Gubler DJ, 1987. Detecting artificial antidengue IgM immune complexes using an enzyme-linked immunosorbent assay. Am J Trop Med Hyg 36: 153-159.

33. Chungue E, Marche G, Plichart R, Boutin JP, Roux J, 1989. Comparison of immunoglobulin $\mathrm{G}$ enzyme-linked immunosorbent assay (IgG-ELISA) and haemagglutination inhibition (HI) test for the detection of dengue antibodies. Prevalence of dengue IgG-ELISA antibodies in Tahiti. Trans $R$ Soc Trop Med Hyg 83: 708-711.

34. Gubler DJ, Suharyono W, Lubis I, Eram S, Sulianti Saroso J, 1979. Epidemic dengue hemorrhagic fever in rural Indonesia. I. Virological and epidemiological studies. Am J Trop Med Hyg 28: 701-710.

35. Dunnett CW, 1980. Pairwise Multiple Comparisons in the Homogeneous variance unequal sample size case. J Am Stat Assoc 75: 789-795.

36. Rothman KJaG, Sander, 1998. Introduction to Stratified Analysis. Rothman KJ, Sander G, eds. Modern Epidemiology. Philadelphia: Lippincott Williams and Wilkins.

37. Vitarana T, 1982. Viral diseases in Sri Lanka: a national overview. Mackenzie JS, ed. Viral Diseases in South East Asia and the Western Pacific. London: Academic Press, 198-204.

38. Vitarana T, Jayasekera N, 1983. Study of dengue in a low DKF area - Sri Lanka. Pang T, Pathmanathan R, eds. Proceedings of 
the International Conference on Dengue/Dengue Haemorrhagic Fever. Kuala Lumpur, University of Malaya. 103-109.

39. Rico-Hesse R, Harrison LM, Nisalak A, Vaughn DW, Kalayanarooj S, Green S, Rothman AL, Ennis FA, 1998. Molecular evolution of dengue type 2 virus in Thailand. Am J Trop Med Hyg 58: 96-101.

40. Leitmeyer KC, Vaughn DW, Watts DM, Salas R, Villalobos I, de C, Ramos C, Rico-Hesse R, 1999. Dengue virus structural dif- ferences that correlate with pathogenesis. J Virol 73: $4738-4747$.

41. Lewis JA, Chang GJ, Lanciotti RS, Kinney RM, Mayer LW, Trent DW, 1993. Phylogenetic relationships of dengue-2 viruses. Virology 197: 216-224.

42. Lanciotti RS, Lewis JG, Gubler DJ, Trent DW, 1994. Molecular evolution and epidemiology of dengue-3 viruses. J Gen Virol 75: $65-75$. 